\title{
ESTUDO DE CASO DE UM ECOSSISTEMA DE NEGÓCIOS CIRCULAR SOB A PERSPECTIVA DE UMA EMPRESA ORQUESTRADORA
}

Ana Hiromi Shimozono (anahiromi@ usp.br) - Escola de Engenharia de São Carlos, Universidade de São Paulo (USP).

Adriana Hofmann Trevisan (마rianatrevisan@usp.br) - Escola de Engenharia de São Carlos, Universidade de São Paulo (USP).

Camila Gonçalves Castro (camilagcastro@usp.br) - Escola de Engenharia de São Carlos, Universidade de São Paulo (USP).

Janaina Mascarenhas (jana.mascarenhas@usp.br) - Escola de Engenharia de São Carlos, Universidade de São Paulo (USP).

\section{RESUMO}

A economia circular (EC) visa minimizar o impacto ambiental causado durante toda a cadeia produtiva, podendo ser alcançada por meio da implementação de estratégias circulares e da colaboração entre diferentes atores em um ecossistema de negócios. Apesar da íntima relação entre os conceitos de EC e ecossistemas de negócios, poucos trabalhos relacionando ambos os temas foram encontrados. Este trabalho, portanto, procura contribuir ao estudar um ecossistema de reciclagem de embalagens longa-vida, sob a perspectiva de uma empresa orquestradora no Brasil. Foi selecionado o método de estudo de caso exploratório para identificar o estágio de vida atual em que o ecossistema se encontra, os atores envolvidos e seus relacionamentos, assim como as principais estratégias implementadas para viabilizar a reciclagem de embalagens. A partir da análise de dados, obtidos através de entrevistas e relatórios de sustentabilidade, verificou-se que a presença de vários atores é fundamental para fomentar continuamente a reciclagem, além do papel do orquestrador em gerenciar os diversos interesses dentro de um ecossistema, de forma a guiá-lo para uma maior circularidade. Por fim, este trabalho procura contribuir para gerar discussões e estudos futuros sobre ecossistema de negócios e EC, principalmente no contexto brasileiro, onde há poucas evidências de pesquisa nesse ramo.

Palavras-chave: ecossistema de negócios; economia circular; reciclagem; embalagem. 


\section{INTRODUÇÃO}

A implementação de um novo modelo econômico mais sustentável vem sendo discutido entre estudiosos, empresários e formuladores de políticas, dado quão consumista e predatório é o atual modelo linear (GEISSDOERFER et al., 2017). A economia circular (EC) procura garantir essa maior sustentabilidade, uma vez que visa manter os materiais o máximo de tempo possível nos ciclos, sempre explorando o seu mais alto nível de valor e utilidade (EMF, 2015). A EC procura minimizar o desperdício, o uso geral de recursos e a emissão de gases (GEISSDOERFER et al., 2017). A circularidade pode ser alcançada por meio da adoção de estratégias circulares, como por exemplo, as elucidadas por Potting et al. (2017): R0. Recusar, R1. Repensar, R2. Reduzir, R3. Reutilizar, R4. Reparar, R5. Recondicionar, R6. Remanufaturar, R7. Reaproveitar, R8. Reciclar e R9. Recuperar. Além dessas estratégias, outro fator importante, observado em casos de sucesso na transição para uma EC, foi o envolvimento de diferentes atores e da capacidade dos mesmos em estabelecer uma relação de colaboração (GHISELLINI; CIALANI; ULGIATI, 2016).

Diferentes atores relacionando entre si para alcançar um resultado em comum (GEISSDOERFER et al., 2017), atuando como membros de uma rede e não como entidades isoladas, remete ao conceito de ecossistema de negócios (TATE et al., 2019). Inicialmente definido por Moore (1993), um ecossistema de negócios, assim como um ecossistema biológico, depende de vários organismos para prosperar e se tornar uma comunidade mais estruturada.

Na literatura, há estudos integrando e disseminando os conceitos de ecossistema de negócios e EC, uma vez que a transição para o novo modelo econômico requer a colaboração de diferentes stakeholders. Os artigos de De Langen, Sornn-Friese e Hallworth (2020) e Hsieh et al. (2017) ilustram a integração dessas duas temáticas, dado que o primeiro analisou o pioneirismo circular de um ecossistema portuário de Amsterdã e, o segundo identificou os mecanismos que permitiram a liderança de uma empresa no ecossistema de reciclagem de vidro em Taiwan.

Neste artigo, por sua vez, foi estudado um ecossistema de negócios de reciclagem de embalagens longa-vida a partir da perspectiva do orquestrador, através do mapeamento dos atores envolvidos e de seus relacionamentos. Foram identificadas também as estratégias circulares implementadas, assim como o atual estágio de vida do ecossistema. A principal 
motivação para a condução deste estudo foi a importância de elucidar como os ecossistemas vêm adotando práticas circulares, a fim de garantirem sua sobrevivência e competitividade.

Este trabalho fornece uma contribuição à teoria ao analisar como uma empresa é capaz de orquestrar o ecossistema o qual lidera, ambientado no contexto brasileiro, onde ainda há poucos registros de artigos nessa área. E à prática, uma vez que permite que outras empresas aproveitem esses registros para futuros insights, adaptando-os de acordo com a sua realidade.

\section{REVISÃO TEÓRICA}

\subsection{Orquestradores em ecossistemas de negócios}

Um ecossistema de negócios é composto por vários atores que podem ser identificados como sendo as próprias empresas e seus consumidores, fornecedores e parceiros (MOORE, 1993). Cada membro desempenha uma função distinta e, juntos, têm como objetivo criar um produto ou serviço que constitua uma solução coerente a todos os envolvidos (PIDUN; REEVES; SCHÜSSLER, 2019).

Moore (1993) explicitou algumas particularidades que facilitam a identificação dos quatro estágios do ciclo de vida do ecossistema, sendo elas: (i) nascimento - as empresas co-evolutivas determinam um produto ou serviço que melhor atenda às necessidades dos consumidores; (ii) expansão - dominação de uma porcentagem maior de mercado; (iii) liderança - as empresas possuem perspectivas futuras, encorajando seus stakeholders a continuarem com a parceria e (iv) auto-renovação ou morte - caracterizado pelo trabalho com outros ecossistemas ou com inovações que venham a surgir dentro de seu próprio ecossistema.

Apesar da presença fundamental de cada membro, possibilitando uma maior resiliência devido ao acesso a uma ampla gama de capabilidades (PIDUN; REEVES; SCHÜSSLER, 2019), Parida et al. (2019) destacam o papel dos orquestradores na transição para uma EC. Os orquestradores são responsáveis por promover a saúde geral do ecossistema, ao facilitar a comunicação entre os stakeholders e ao criar e compartilhar valor (IANSITI; LEVIEN, 2004). Em suma, os orquestradores devem gerenciar os diversos interesses e assegurar o alinhamento entre os parceiros (PARIDA et al., 2019).

Um ecossistema que adota a EC apresenta vantagens competitivas, dado o seu potencial de gerar valor, devido: (i) à capacidade de produzir com menores custos; (ii) ao aumento do ciclo de vida dos produtos; (iii) ao poder do uso em cascata e (iv) ao poder dos próprios círculos, 
onde os materiais permanecem sem contaminação, aumentando, portanto, a eficiência e a produtividade material (MURRAY; SKENE; HAYNES, 2017).

\subsection{Estratégias que viabilizam a transição para uma economia circular}

A EC pode operar em três níveis, sendo eles: micro (empresas e consumidores), meso (ecossistemas e parques eco-industriais) e macro (nações e formuladores de políticas) (KIRCHHERR; REIKE; HEKKERT, 2017; VALKOKARI et al., 2014). Uma vez operada nestes três níveis, a EC tem a capacidade de promover um desenvolvimento sustentável, garantindo beneficiar tanto a sociedade e o meio ambiente, quanto a economia (KIRCHHERR; REIKE; HEKKERT, 2017).

Apesar de existirem muitos trabalhos propondo diferentes abordagens para as estratégias "R", resultando em diversos frameworks, essas estratégias se assemelham em termos de funcionalidade, ou seja, podem ser compreendidas como princípios de operacionalização da economia circular (REIKE; VERMEULEN; WITJES, 2018). Alguns exemplos desses frameworks são os 3R (reduzir, reutilizar e reciclar) descritos no artigo de Sakai et al. (2011), os 6R de Yan e Feng (2014) que acrescentam as estratégias recuperar, remanufaturar e redesign e, ainda, os 9R de Potting et al. (2017), os quais serão utilizados neste estudo devido à sua maior abrangência.

A Tabela 1 fornece uma breve descrição dos $9 \mathrm{R}$, sendo que as mesmas foram organizadas em ordem decrescente de circularidade, ou seja, a estratégia "recusar" apresenta a maior circularidade e, portanto, garante uma menor utilização de matéria-prima e uma menor pressão ambiental (POTTING et al., 2017). 
TABELA 1 - Descrição das estratégias 9R

\begin{tabular}{|c|c|c|c|}
\hline $\mathbf{R}$ & Estratégia & Descrição & Referências \\
\hline 0 & Recusar & $\begin{array}{l}\text { Recusa a adquirir novos produtos e a utilizar } \\
\text { processos tóxicos ou que geram muito } \\
\text { desperdicio. }\end{array}$ & $\begin{array}{l}\text { REIKE; VERMEULEN; WITJES } \\
(2018)\end{array}$ \\
\hline 1 & Repensar & $\begin{array}{l}\text { Garantia do uso mais intensivo do produto por } \\
\text { meio do compartilhamento e exploração do valor } \\
\text { residual dos produtos. }\end{array}$ & $\begin{array}{l}\text { POTTING et al., (2017) e } \\
\text { BLOMSMA et al., (2019) }\end{array}$ \\
\hline 2 & Reduzir & $\begin{array}{l}\text { Redução do impacto ambiental através de uma } \\
\text { produção mais eficiente, a qual pode ser obtida } \\
\text { através de tecnologias mais avançadas. }\end{array}$ & $\begin{array}{l}\text { GHISELLINI; CIALANI; } \\
\text { ULGIATI (2016) }\end{array}$ \\
\hline 3 & Reutilizar & $\begin{array}{l}\text { Qualquer operação em que os componentes ou } \\
\text { produtos não desperdiçados são utilizados } \\
\text { novamente com o mesmo propósito de quando } \\
\text { foram manufaturados. }\end{array}$ & $\begin{array}{l}\text { CASTELLANI; SALA; } \\
\text { MIRABELLA (2015) }\end{array}$ \\
\hline 4 & Reparar & $\begin{array}{l}\text { Correção de falhas em componentes especificos } \\
\text { para que os produtos possam atender às funções } \\
\text { originais, expandindo o ciclo de vida dos } \\
\text { mesmos. }\end{array}$ & $\begin{array}{c}\text { POTTING et al. (2017) e KING et } \\
\text { al. (2006) }\end{array}$ \\
\hline 5 & Recondicionar & $\begin{array}{l}\text { Processo de tornar um produto obsoleto em um } \\
\text { satisfatório para os clientes, apesar de apresentar } \\
\text { uma qualidade inferior ao produto original. }\end{array}$ & $\begin{array}{l}\text { DEN HOLLANDER; BAKKER E } \\
\text { HULTINK (2017) e KING et al. } \\
(2006)\end{array}$ \\
\hline 6 & Remanufaturar & $\begin{array}{l}\text { Utilização de componentes de produtos antigos } \\
\text { para a fabricação de novos ou para a reparação de } \\
\text { defeituosos, atendendo às mesmas expectativas } \\
\text { dos consumidores que um produto novo. }\end{array}$ & $\begin{array}{c}\text { POTTING et al. (2017) e KING et } \\
\text { al. (2006) }\end{array}$ \\
\hline 7 & Reaproveitar & $\begin{array}{l}\text { Utilização de componentes velhos para a } \\
\text { fabricação de produtos novos com funções } \\
\text { completamente novas. }\end{array}$ & $\begin{array}{l}\text { REIKE; VERMEULEN; WITJES } \\
(2018)\end{array}$ \\
\hline 8 & Reciclar & $\begin{array}{l}\text { Processamento de materiais para obtenção de um } \\
\text { produto com a mesma ou com uma qualidade } \\
\text { inferior. }\end{array}$ & POTTING et al. (2017) \\
\hline 9 & Recuperar & $\begin{array}{l}\text { Recuperação de energia por meio de incineração, } \\
\text { pirólise ou digestão anaeróbica. }\end{array}$ & $\begin{array}{l}\text { REIKE; VERMEULEN; WITJES } \\
\text { (2018) }\end{array}$ \\
\hline
\end{tabular}

Fonte: Baseada em Potting et al. (2017)

Trabalhos como o de Okorie et al. (2018) e de Ang et al. (2021) exemplificam a importância e a aplicação das estratégias 9R, tanto para acadêmicos e formuladores de política, quanto para empresários. O primeiro estudou como a integração entre tecnologias digitais e a implementação de práticas circulares, as $9 \mathrm{R}$, apoiam a indústria 4.0, permitindo a transição para uma EC. Já o segundo, baseado em Potting et al. (2017), propôs um framework, reforçando a implementação das 9 estratégias circulares no setor de fabricação de produtos farmacêuticos.

Para este trabalho, tanto as estratégias quanto os estágios de vida descritos anteriormente serão utilizados para identificar as práticas circulares implementadas pela empresa e a evolução 
do ecossistema. Além disso, será analisado o papel do orquestrador dentro do ecossistema, em função de esse ator ser frequentemente destacado pela literatura como um dos principais membros (PARIDA et al., 2019).

\section{METODOLOGIA}

Este artigo utilizou o método de estudo de caso exploratório, dada a possibilidade de investigar, em profundidade, um fenômeno de interesse e o seu contexto (RIDDER, 2017). Além disso, o método permite compreender um conjunto de decisões a partir de questionamentos como: por que essas decisões foram tomadas, como foram implementadas e qual o resultado final (YIN, 2003). Este estudo foi inspirado no trabalho de Hsieh et al. (2017), o qual analisou a empresa Spring Pool Glass, líder em reciclagem de vidro em Taiwan, que atualmente governa seu ecossistema de negócios. Os autores descreveram os estágios de vida do ecossistema, além de elucidar as principais práticas que permitiram a posição de liderança da empresa.

De maneira similar, neste estudo, o objeto de interesse foi o ecossistema de negócios de reciclagem de embalagens longa-vida, o qual foi estudado a partir do ponto de vista de uma empresa orquestradora. A empresa, fabricante de embalagens longa-vida e de origem sueca, foi fundada em 1951 e se instalou no Brasil em 1957, onde atualmente possui duas fábricas: a primeira inaugurada em 1978 e a segunda em 1999.

Para a coleta de dados, foram realizadas entrevistas e análises de dados secundários, sendo estes alguns dos métodos mais utilizados em estudos de casos (EISENHARDT, 1989). Em um primeiro momento, foram analisados os relatórios anuais de sustentabilidade de 2018, 2019 e 2020, disponíveis no site oficial da empresa, a fim de identificar, principalmente, as estratégias circulares adotadas.

Em um segundo momento, foram realizadas entrevistas com um analista de sustentabilidade (entrevistado 1), que ocupa este cargo há 9 meses, e um gerente de sustentabilidade (entrevistado 2), que atua na empresa há mais de 10 anos. Com o consenso dos participantes, as entrevistas foram transcritas para que a perda de conteúdo fosse a mínima possível. Além disso, foram adotadas questões abertas, dada a pouca quantidade de entrevistados, o que permitiu maiores discussões e aprofundamentos, conforme o nível de detalhamento fornecido pelos mesmos. 
Durante as entrevistas, foram levantadas questões relacionadas ao histórico da empresa, à rede de stakeholders e seus relacionamentos, à percepção da empresa sobre EC e suas práticas sustentáveis, bem como sobre as principais dificuldades enfrentadas atualmente em termos de circularidade. Os resultados obtidos, através da análise das entrevistas e dos relatórios de sustentabilidade, são apresentados no tópico a seguir.

\section{RESULTADOS E DISCUSSÃO}

\subsection{Entendendo a evolução do ecossistema sob a perspectiva do orquestrador}

Analisando as entrevistas realizadas e o market share da empresa, verificou-se que o estágio de nascimento, caracterizado pela definição da proposta de valor (MOORE, 1993), se iniciou em 1951, quando a empresa foi fundada na Suécia com o propósito de transportar alimentos em embalagens seguras. Esse período se encerrou em 1957, quando a empresa iniciou suas atividades no Brasil, dando-se início ao próximo estágio, o de expansão, como ilustra a Figura 1.

FIGURA 1 - Histórico do Market Share da empresa no Brasil.

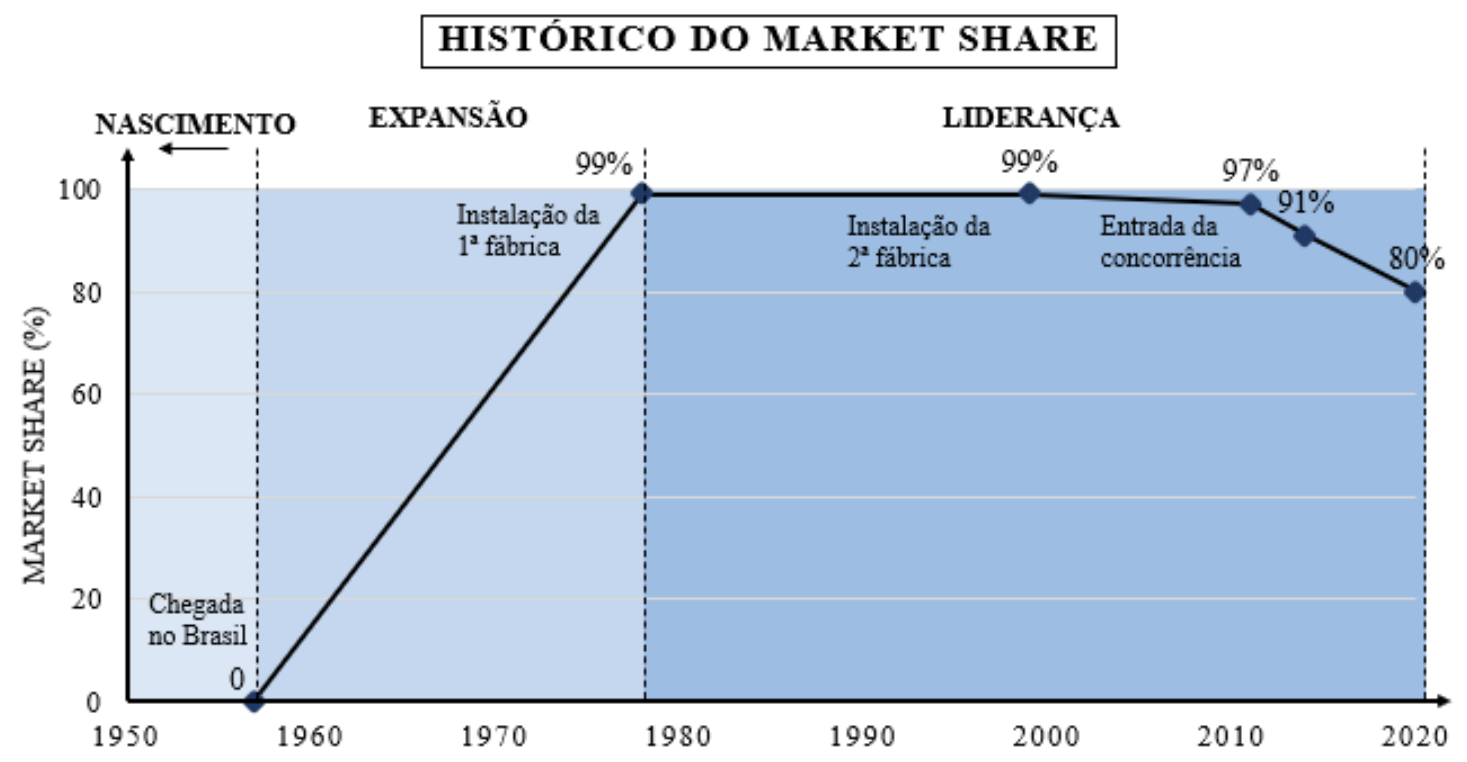

Fonte: Os autores

O segundo estágio, caracterizado pela expansão no market share (HSIEH et al., 2017), foi observado entre 1957 e 1978, quando a empresa instalou a sua primeira fábrica no interior do Estado de São Paulo. Nesse período, a empresa viu seu market share crescer rapidamente, apesar de a fábrica ainda produzir embalagens em escalas pequenas. Esse crescimento se deu, principalmente, pela ausência de um grande competidor na época, o que permitiu a rápida ascensão da empresa. 
Já o terceiro e atual estágio, o de liderança, é caracterizado pelo controle do ecossistema (MOORE, 1993), principalmente em função da alta porcentagem de market share, muito próximo dos $99 \%$, a partir dos anos 80 . Nesse período de liderança, verificou-se a inauguração da segunda fábrica em 1998, dada a necessidade de atender o aumento da demanda do mercado brasileiro.

No entanto, a partir dos anos 2010, a empresa viu seu market share cair de $2 \%$ a $3 \%$ ao ano, dada a entrada de concorrentes. Apesar desse decaimento, a empresa ainda mantém sua posição de líder e orquestradora do ecossistema, visto que a mesma não se limitou à fabricação e à venda de embalagens, mas expandiu seu mercado para o fornecimento de soluções ponta a ponta, desde a produção da embalagem e vazamento do produto, até a solução para produção de alimentos. Esse diferencial permitiu que a empresa controlasse uma gama maior de elementos essenciais para o desenvolvimento dos demais atores da rede, garantindo seu alto poder de barganha.

\subsection{Os diferentes atores e seus relacionamentos}

Durante as entrevistas, foram feitas perguntas relacionadas à rede de atores, os quais foram divididos em fornecedores, clientes, consumidores finais e parceiros, e aos relacionamentos mantidos com os mesmos, sendo eles de cooperação e competição.

Sob a perspectiva da empresa orquestradora, fabricante de embalagens, os entrevistados listaram seus três maiores fornecedores de matérias-primas, sendo eles de plástico, papel e alumínio. Eles ressaltaram que, por conta das rígidas especificações, empresas de menor porte tendem a não atingir todos os critérios necessários para a garantia da segurança alimentar nas embalagens.

Conforme indicado na Figura 2, a empresa estudada mantém uma relação cooperativa com todos os atores envolvidos no ecossistema, no entanto, verifica-se que com os fornecedores e os clientes, a competitividade também está presente. Essa competição tem como causa principal a retenção de tecnologias únicas por parte dos fornecedores, o que lhes garantem um alto poder de barganha sobre os demais membros do ecossistema, incluindo a empresa estudada. 
FIGURA 2 - Mapeamento dos atores sob a perspectiva do orquestrador.

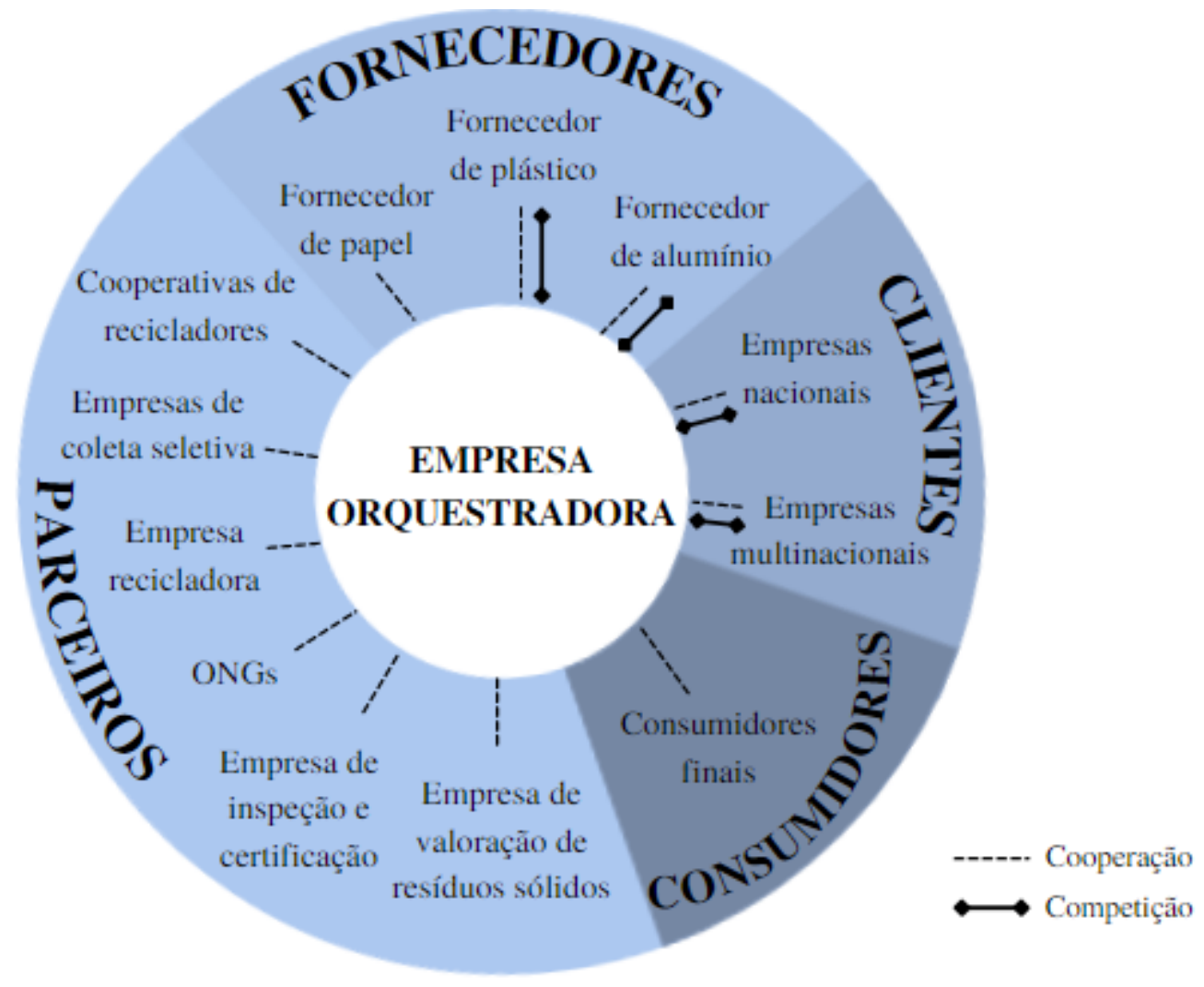

Fonte: Os autores

Já entre os clientes, representados pelas empresas nacionais e multinacionais que adquirem os produtos para embalar os alimentos, um exemplo de competição notado foi o desenvolvimento de tecnologias de fabricação de embalagens, muito similares às fornecidas pela empresa estudada. Para os clientes, esse desenvolvimento é benéfico por representar uma maior independência em relação à empresa fabricante, enquanto que, para a última, essa independência representa uma perda de mercado.

Os consumidores finais das embalagens longa-vida possuem papel fundamental dentro do ecossistema, uma vez que, após o consumo, realizam o descarte desses produtos. Em seguida, têm-se os parceiros que atuam ativamente para fomentar o valor das embalagens longa-vida. Para a coleta e a reciclagem de embalagens, os entrevistados comentaram sobre a importância de a empresa orquestradora fornecer um know-how e desenvolver equipamentos apropriados para a reciclagem, de forma a viabilizar o trabalho desenvolvido pelas cooperativas de recicladores e empresas de coleta seletiva. O material reciclado, oriundo da coleta seletiva, é então utilizado por empresas recicladoras que fabricam novos produtos, como termoplásticos e telhas, aumentando o valor agregado. 
As ONGs, por sua vez, atuam em conjunto por meio de projetos sociais voltados para comunidades carentes, onde não há, muitas vezes, acesso à coleta seletiva. Para os locais onde já existe o descarte destinado a embalagens longa-vida, a empresa parceira de certificação e inspeção desenvolveu um aplicativo para informar aos consumidores finais onde há pontos de coleta próximos a eles. Por fim, a empresa de valoração de resíduos desenvolveu, através da implementação da tecnologia blockchain, uma plataforma que permite conectar interessados na compra e venda de resíduos, como as embalagens longa-vida.

\subsection{Estratégias circulares adotadas pela empresa orquestradora}

Como o foco do ecossistema estudado são as embalagens longa-vida no Brasil, foram identificadas algumas estratégias referentes a esse produto, obtidas através dos relatórios anuais de sustentabilidade e das entrevistas. Dentre as estratégias 9R de Potting et al. (2017), 3 estão diretamente associadas às embalagens: 1. Reciclagem das embalagens através de equipamentos fornecidos pela própria empresa; 2. Reaproveitamento do plástico-alumínio retirado das embalagens e 3.Recusa a utilização de materiais de fontes não-renováveis para a fabricação de produtos.

Reciclagem: De acordo com o entrevistado 2: “(...) houve um desenvolvimento tecnológico, investimento em equipamentos e testes laboratoriais para ter esse processo [de reciclagem] feito". Como a tecnologia para separação das fibras de papelão era previamente conhecida, a empresa a adaptou, a fim de realizar a desagregação do plástico-alumínio, utilizado no interior das embalagens, garantindo um material final mais limpo e de melhor qualidade.

Reaproveitamento: A parceria com uma empresa recicladora permitiu que o plásticoalumínio retirado das embalagens fosse transformado em um material próprio para a fabricação de pellets, telhas e chapas ecológicas. O reaproveitamento, além de garantir um aumento no valor agregado, incentiva o desenvolvimento de um novo mercado, o qual, segundo o entrevistado 2: "representa praticamente 90\% desse mercado de plástico-alumínio".

Recusa: A empresa passou a incorporar o uso de polietileno oriundo da cana-de-açúcar, substituindo aquele de fontes não-renováveis, para a fabricação de tampas e camadas de filme das embalagens. O polietileno renovável possui as mesmas características que um plástico comum, podendo ser reciclado pelas cadeias recicladoras já existentes, ao mesmo tempo em que reduz a emissão de gases poluentes, dada a captação de $\mathrm{CO} 2$ realizada na própria cadeia produtiva. 
Apesar da implementação de algumas estratégias, como as apontadas anteriormente, existem desafios que dificultam o ecossistema a se tornar cada vez mais circular, sendo eles: (i) a legislação brasileira atual ainda é branda quanto à fiscalização, o que acaba desincentivando empresas a adotarem o modelo circular; (ii) a falta de conscientização sobre o consumo e descarte correto, assim como a falta de acesso à coleta seletiva por grande parte da população e (iii) a resistência de algumas empresas em adotar o design for environment, no qual os produtos são projetados de forma a causarem o menor impacto ambiental possível.

\section{CONCLUSÃO}

Este estudo buscou compreender um ecossistema de reciclagem de embalagens longa-vida, através da perspectiva do orquestrador. Foram analisados a evolução do ecossistema, os atores envolvidos e suas relações, bem como as estratégias implementadas pela empresa.

Verificou-se, portanto, que a empresa estudada atualmente ocupa uma posição de liderança dentro do ecossistema, em função, principalmente, do seu diferencial em fornecer não apenas embalagens, mas também soluções de ponta a ponta a seus clientes. Seu papel orquestrador se mostrou essencial para o gerenciamento dos interesses de diversos atores, assim como para o fornecimento de capacitação (know-how) a outros parceiros. A presença e atuação de vários membros se mostrou uma característica fundamental para fomentar continuamente o valor da reciclagem de embalagens. Além disso, as estratégias implementadas demonstraram que existe uma grande preocupação, por parte da empresa, em diminuir o impacto ambiental gerado em toda a cadeia produtiva. No entanto, ainda existem barreiras nos âmbitos social, governamental e empresarial que dificultam a transição para uma economia cada vez mais circular.

É importante ressaltar que este trabalho utilizou o estudo de caso único. Esse método, apesar de possibilitar o entendimento mais aprofundado sobre o objeto em questão, impede que generalizações sejam feitas. Além disso, os dados primários foram obtidos através de entrevistas com apenas dois funcionários do setor de sustentabilidade, o que acaba representando uma limitação por apenas retratar a visão exclusiva do orquestrador.

No entanto, espera-se que, a partir deste estudo, novas pesquisas possam ser conduzidas e que ecossistemas que desejem adotar a economia circular possam ter novos insights, adaptandoos para suas realidades. 


\section{AGRADECIMENTOS}

À Fundação de Amparo à Pesquisa do Estado de São Paulo (FAPESP), referente aos processos $n^{\circ} 2020 / 15831-9$ e nº 2019/23655-9, por todo apoio financeiro. É importante ressaltar que as opiniões, hipóteses e conclusões ou recomendações expressas neste material são de responsabilidade dos autores e não necessariamente refletem a visão da FAPESP.

\section{REFERÊNCIAS}

ANG, K. L. et al. Sustainability framework for pharmaceutical manufacturing (PM): a review of research landscape and implementation barriers for circular economy transition. Journal of Cleaner Production, v. 280, 2021. DOI: https://doi.org/10.1016/j.jclepro.2020.124264.

BLOMSMA, F. et al. Developing a circular strategies framework for manufacturing companies to support circular economy-oriented innovation. Journal of Cleaner Production, v. 241, 2019. DOI: https://doi.org/10.1016/j.jclepro.2019.118271.

CASTELLANI, V.; SALA, S.; MIRABELLA, N. Beyond the throwaway society: a life cyclebased assessment of the environmental benefit of reuse. Integrated Environmental Assessment and Management, v. 11, n. 3, p. 373-382, 2015.

DE LANGEN, P. W.; SORNN-FRIESE, H.; HALLWORTH, J. The Role of port development companies in transitioning the port business ecosystem; the case of port of Amsterdam's circular activities. Sustainability (Switzerland), v. 12, n. 11, p. 1-16, 2020.

DEN HOLLANDER, M. C.; BAKKER, C. A.; HULTINK, E. J. Product design in a circular economy: development of a typology of key concepts and terms. Journal of Industrial Ecology, v. 21, n. 3, p. 517-525, 2017.

EISENHARDT, K. M. Building theories from case study research. Academy of Management Review, v. 14, n. 4, p. 532-550, Oct. 1989. DOI: https://doi.org/10.2307/258557.

ELLEN MACARTHUR FOUNDATION. Rumo à economia circular: o racional de acelerar a transição. [S.1]: Ellen MacArthur Foundation, 2015, p. 22.

GEISSDOERFER, M. et al. The Circular economy - a new sustainability paradigm? Journal of Cleaner Production, v. 143, p. 757-768, 2017.

GHISELLINI, P.; CIALANI, C.; ULGIATI, S. A Review on circular economy: the expected transition to a balanced interplay of environmental and economic systems. Journal of Cleaner Production, v. 114, p. 11-32, 2016.

HSIEH, Y. C. et al. Governing a sustainable business ecosystem in Taiwan's circular economy: the story of spring pool glass. Sustainability (Switzerland), v. 9, n. 6, 2017. DOI: http://doi.org/10.3390/su9061068.

IANSITI, M.; LEVIEN, R. Strategy as ecology. Harvard Business Review, v. 82, n. 3, 2004. 
KING, A. M. et al. Remanufacture or recycle? Sustainable Development, v. 14, p. 257-267, Dec. 2006.

KIRCHHERR, J.; REIKE, D.; HEKKERT, M. Conceptualizing the circular economy: an analysis of 114 definitions. Resources, Conservation and Recycling, v. 127, p. 221-232, Ap. 2017.

MOORE, J. F. A New ecology of competition Harvard BusinessReview. Harvard Business Review, v. 71, n. 3, p. 75-86, 1993.

MURRAY, A.; SKENE, K.; HAYNES, K. The Circular economy: an interdisciplinary exploration of the concept and application in a global context. Journal of Business Ethics, v. 140, n. 3, p. 369-380, 2017.

OKORIE, O. et al. Digitisation and the circular economy: a review of current research and future trends. Energies, v. 11, n. 11, p. 1-31, 2018.

PARIDA, V. et al. Orchestrating industrial ecosystem in circular economy: a two-stage transformation model for large manufacturing companies. Journal of Business Research, v. 101, p. 715-725, Aug. 2019.

PIDUN, U.; REEVES, M.; SCHÜSSLER, M. Do you need a business ecosystem? [S.1.]: BCG Henderson Institute, 2019, p.11.

POTTING, J. et al. Circular economy: measuring innovation in the product chain - policy report. Hague: PBL Netherlands Environmental Assessment Agency, 2017, p.42. (PBL publication number, 2544).

REIKE, D.; VERMEULEN, W. J. V.; WITJES, S. The circular economy: new or refurbished as CE 3.0? - exploring controversies in the conceptualization of the circular economy through a focus on history and resource value retention options. Resources, Conservation and Recycling, v. 135, p. 246-264, Aug. 2018.

RIDDER, H. G. The Theory contribution of case study research designs. Business Research, v. 10, n. 2, p. 281-305, 2017.

SAKAI, S. I. et al. International comparative study of 3R and waste management policy developments. Journal of Material Cycles and Waste Management, v. 13, n. 2, p. 86-102, 2011.

TATE, W. L. et al. Seeing the forest and not the trees: learning from nature's circular economy. Resources, Conservation and Recycling, v. 149, p. 115-129, 2019.

VALKOKARI, K. et al. Road-mapping the business potential of sustainability within the European Manufacturing Industry. v. 16, n. 4, p. 360-384, 2014.

YAN, J.; FENG, C. Sustainable design-oriented product modularity combined with 6R concept: a case study of rotor laboratory bench. Clean Technologies and Environmental Policy, v. 16, 
13ำ Congresso Brasileiro de Inovação e Gestão de Desenvolvimento do Produto

08 a 10 de setembro de 2021 - Escola de Engenharia de \$ão Carlos - Universidade de \$ão Paulo

n. 1, p. 95-109, 2014.

YIN, R. K. Case study research - design and methods. Sage: Los Angeles, 2003. 